\title{
A Minimal Factor Overlap Method for Resolving Ambiguity in Factor Analysis of Dynamic Cardiac PET
}

\author{
R. Klein, M. Bentourkia, R.S. Beanlands, A. Adler, R. deKemp
}

\begin{abstract}
Factor analysis has been pursued as a means to decompose dynamic cardiac PET images into different tissue types based on their unique physiology. Each tissue is represented by a time-activity profile (factor) and an associated spatial distribution (structure). Decomposition is based on non-negative constraints of both the factors and structures; however, additional constraints are required to achieve a unique solution. In this work we present a novel method (minimal factor overlap - MFO) and compare its performance to a previously published constraint (minimal spatial overlap - MSO). We compared both methods using simulated data and on a canine model with different ${ }^{82} \mathrm{Rb}$ infusion profiles. Biasing of factors due to spillover is reduced with MFO compared to MSO, while the robustness and reproducibility of MSO is maintained.
\end{abstract}

\section{INTRODUCTION}

$\mathrm{F}$ actor analysis techniques have been explored as a means to improve cardiac function quantification. An image series is decomposed into a finite number of temporal factors and their corresponding spatial distribution (structures) which ideally should correspond to the physiology of the imaged tissue [1]. The decomposition may be expressed in matrix form as

$$
Y=F S+E \text {, }
$$

Where $\mathrm{Y}$ is the dynamic image sequence (the pixels of each time frame in a row), the columns of $F$ contain the time-activity profiles of the factors, the rows of S contain spatial distribution (structure) of the factor, and $\mathrm{E}$ is error.

Decomposition is non-unique, requiring constraints that model the physical imaging process. In cardiac PET, these have historically been decomposition into non-negative factors and structures, which is representative of the physics and imaging process. In addition, Poisson statistics have been used to model the imaging process, but these constraints still do not ensure a unique solution.

In 2006 El Fahkri et al. [2] introduced an additional constraint that minimizes structure overlap in order to ensure a unique solution. This served their purpose of extracting blood timeactivity-curves using the LV blood factor. In this work we propose an alternative constraint that minimizes factor overlap, in order to improve the physiological accuracy of the factors and associated structures.

\section{METHODS AND MATERIALS}

Two sets of data were analyzed:

1. A simulated dynamic image sequence containing two factors. The first region was a centered circle containing $100 \%$ blood. The second region was a centered ring containing $80 \%$ myocardium and $20 \%$ blood factors. Each time frame of the simulated data was smoothed with a $12 \mathrm{~mm}$ FWHM Gaussian filter resulting in an image containing factors as shown in leftmost column (Source) of table 1.

2. A single dog that underwent a series of dynamic PET scans with varying ${ }^{82} \mathrm{Rb}(150 \mathrm{MBq})$ infusion durations $(15,30,60$, $120,240,240,120,60,30,15$ seconds) with a Siemens ECAT ART scanner. The images were iteratively reconstructed to $12 \mathrm{~mm}$ resolution.

These data sets were analyzed using the following fully automated steps:

1. Cropping of field-of-view to include regions of high signal intensity.
2. The number of factors was determined using the cumulative eigenvalues of the correlation matrix.

3. Factor analysis of medical image sequences (FAMIS) was applied with a relaxed non-negativity constraint as proposed in [1] (98\% non-negative confidence interval). Resulting in factors $F^{\prime}$ and structures $S^{\prime}$.

4. The ambiguity of the solution was resolved by iteratively solving for the factor rotation square matrix, $R$, so as to minimize a cost function $f_{\text {tot }}$ in two ways:

a. MSO - Minimal Structure Overlap as described in [2] using the following equation:

$$
f_{\text {tot }}^{M S O}=f_{n}\left(F^{\prime} R, R^{-1} S^{\prime}\right)+0.01 f_{\text {uni }}\left(R^{-1} S^{\prime}\right)
$$

b. MFO - Minimal Factor Overlap using the following:

$$
f_{\text {tot }}^{M F O}=f_{n}\left(F^{\prime} R, R^{-1} S^{\prime}\right)+0.01 f_{u n i}\left(\left(F^{\prime} R\right)^{T}\right)
$$

$f_{n}(F, S)$ is a combined penalty for negative values in both the rotated factors $\left(F=F^{\prime} R\right)$ and rotated structures $\left(S=R^{-1} S^{\prime}\right)$ as described in [2]. $f_{\text {uni }}(\mathrm{X})$ is a penalty for overlap between the rows of $X$, also as described in [2].

\section{RESULTS}

\section{A. Simulation}

The source structures of the two factors in the simulation were nearly exactly recovered using the MFO method, but not with the MSO method. This is most obvious in the blood factors, where the circular pattern is of smaller diameter (table 1).
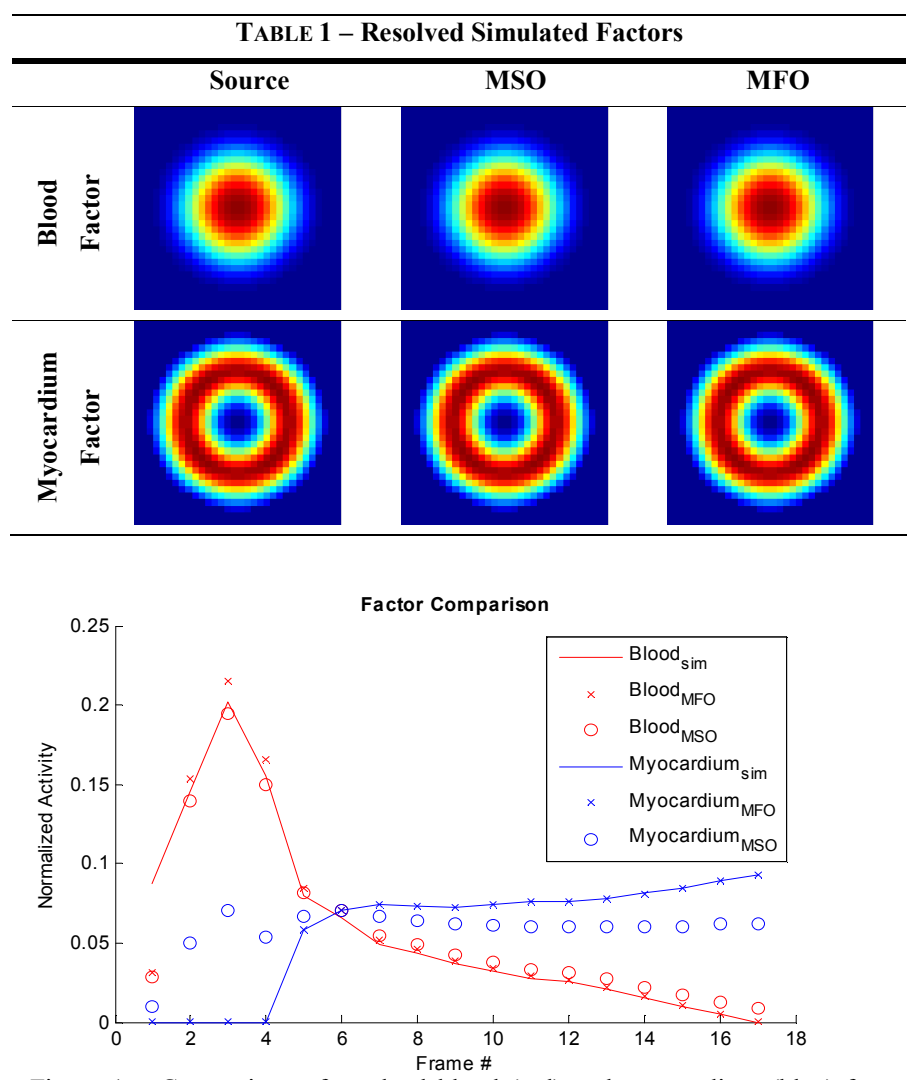

Figure 1 - Comparison of resolved blood (red) and myocardium (blue) factors using MFO (x) MSO (o) to the source profiles (lines) used in simulating the dynamic image sequence. 
Looking at the factor profiles (figure 1) shows that the blood factors using both methods follow the simulated data closely, although MFO appears slightly more accurate $\left(\mathrm{R}^{2}=0.943\right)$ than MSO $\left(\mathrm{R}^{2}=0.927\right)$. With regards to the myocardium factor, MFO was much more accurate $\left(\mathrm{R}^{2}>0.999\right)$ than $\mathrm{MSO}\left(\mathrm{R}^{2}=0.247\right)$.

\section{B. Canine Model}

In all cases 2 factors were automatically determined as sufficient to decompose the image, accounting for $77-91 \%$ of the image variance.

Similarly shaped factors were obtained with both MSO and MFO constraints as demonstrated in figure 2 . The factors were automatically identified (and manually verified) as blood-pool and myocardium.

The myocardium factors obtained with MFO tended to be 'flatter' than those obtained with MSO, i.e. biasing of the myocardium factor with blood (often seen as peak in the myocardium factor in synchrony with the blood pool peak) was reduced using MFO.

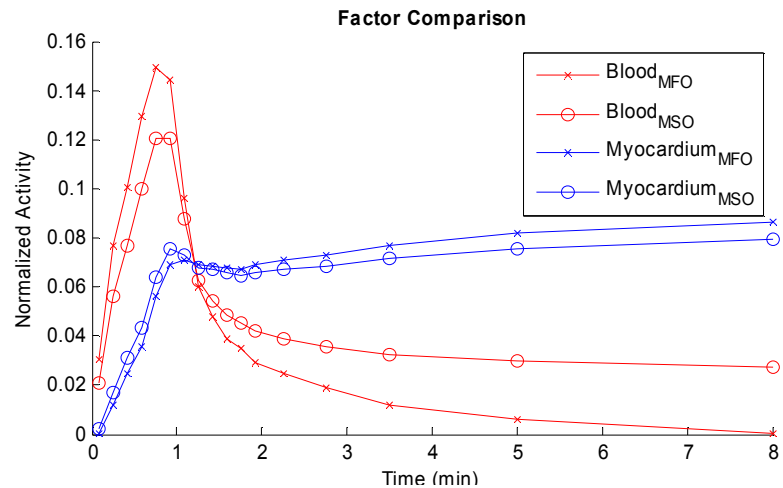

Figure 2 - Example of comparison of resolved blood (red) and myocardium (blue) factors using MFO (x) and MSO (o) in a dog with a 30 second constant activity rate ${ }^{82} \mathrm{Rb}$ infusion.

Using the MFO constraints, the blood factor 'clearance' decreased to nearly zero in the final frames as expected [3], while using MSO they decreased to an asymptote of $15-50 \%$ peak activity, depending on the elution time (Table 2).

\begin{tabular}{lcc}
\hline & $\begin{array}{c}\text { TABLE 2 } \\
\text { (mean of two studies for each elution duration) }\end{array}$ \\
\hline Elution duration & MSO & MFO \\
\hline $15 \mathrm{~s}$ & 0.84 & 1.00 \\
$30 \mathrm{~s}$ & 0.80 & 1.00 \\
$60 \mathrm{~s}$ & 0.76 & 1.00 \\
$120 \mathrm{~s}$ & 0.65 & 1.00 \\
$240 \mathrm{~s}$ & 0.54 & 1.00 \\
\hline
\end{tabular}

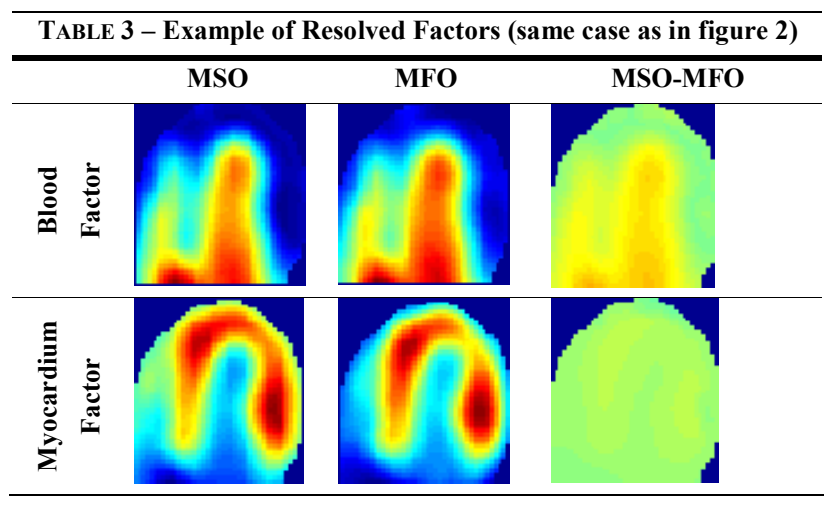

Likewise, the structures obtained using both techniques were similar, as the example in table 3 demonstrates using the same data as in figure 2. The structures using MSO were better resolved, and as expected overlapped less with the myocardium. With MFO more spillover between the structures was observed. Excellent correlation $\left(\mathrm{R}^{2}>0.95\right)$ between structures was measured for all infusion times evaluated, when the same constraint was used, indicating that the results are reproducible using MFO or MSO constraints. Between constraints the correlation was reduced $\left(0.75<\mathrm{R}^{2}<0.87\right)$.

\section{DISCUSSION}

\section{A. Factor Mixing}

The images used in this analysis have been significantly smoothed, increasing the overlap of structures, or bias. When spatial overlap is minimized (MSO), the baseline blood volume in the myocardium is included in the myocardium factor, producing high resolution structures. Conversely, the myocardial spillover into the blood pool becomes included in the blood-pool factor. By reducing the factor overlap, this mixing is discouraged with MFO, which is clearly shown by the results of the simulated data.

\section{B. Number of Factors}

When the images were decomposed into 3 factors the blood-pool and myocardium factors were split to form hybrid factors, supporting the automated selection of 2 factors. The lack of discrimination between LV and RV blood pools in these images indicates that our imaging protocol may lack the temporal resolution required to visualize the transport delay between RV and LV in dogs. On the other hand, this discrimination may not be as important with longer infusion times. Visual inspection of the residue (the portion of the image that is not accounted for by the resolved factors) did not reveal any anatomic structure or persistent temporal pattern. This would indicate that the residue consists primarily of noise, as expected.

\section{Future Work}

The factors and structures should be validated in vivo if possible. It is our intention to compare the blood factors and blood structures to arterial blood sampling and ${ }^{11} \mathrm{CO}$ blood-pool imaging respectively. Absolute myocardial blood flow measurements [4] using these factors and/or structures may also be validated against invasive standards such as microspheres flow.

\section{CONCLUSION}

Constraints must be placed on dynamic cardiac PET image decomposition in order to resolve physiologically accurate factors. Minimizing the overlap between normalized time profiles (factor) overlap provides superior results than those provided by minimizing the spatial overlap of the structures.

\section{REFERENCES}

[1] I. Buvat, H. Benali, R. Di Paola, Statistacal distribution of factors and factor images in factor analysis of medical image sequences, Phys. Med. Biol. 1998;43;1695-1711

[2] G. El Fahkri, A. Sitek, B. Guerin, M. F. Kijewski, M.F. Di Carli, S. C. Moore, Quantitative Dynamic Cardiac 82Rb PET Using Generalized Factor and Compartment Analyses, J. Nuc. Med., 2005;46(8);1264-71

[3] I.N. Weinberg, S.C. Huang, E.J. Hoffman, L. Araujot, C. Nienaber, M. Grover-McKay, M. Dahibom, H. Schelbert, Validation of PET Acquired Input Functions for Cardiac Studies. J. Nuc. Med., 1988;29(2):241-247.

[4] M.Lortie. R.S.B. Beanlands, K. Yoshinaga, R. Klein, J.N. DaSilva, R.A. deKemp, Quantification of Myocardial Blood Flow With ${ }^{82} \mathrm{Rb}$ Dynamic PET Imaging. Eur. J. Nuc. Med. Molec. Imaging, 2007 (in press). 